\title{
Caracterización de una nueva membrana cerámica de microfiltración con soporte de tejido en acero inoxidable flexible
}

\author{
L. PALACIO, P. PRÁDANOS, J. I. CALVO Y A. HERNÁNDEZ \\ Dpto. Termodinámica y Física Aplicada, Facultad de Ciencias, Universidad de Valladolid, 47071 Valladolid, España
}

\begin{abstract}
En este trabajo se ha abordado la caracterización de una membrana cerámica de microfiltración flexible, fabricada por Synthesechemie ${ }^{\circledR}$ con tamaño de poro nominal de $0.45 \mu \mathrm{m}$. Ésta consiste en un soporte poroso de $\alpha$-alúmina que posee un armazón formado por una red metálica de acero inoxidable sobre el cual existe una capa selectiva de óxido de titanio. La caracterización de la membrana ha sido realizada con diversas técnicas como: microscopía de fuerza atómica (AFM), porometría de desplazamiento aire-líquido y porosimetría de mercurio. Cada técnica da información sobre aspectos diferentes: superficie, poros totales y poros activos. Si bien la distribución total de tamaños de poro es bastante ancha, los poros activos (abiertos al flujo) presentan una distribución de tamaños estrecha con un valor medio muy próximo al nominal.
\end{abstract}

Palabras Clave: Materiales porosos, Membranas, Análisis computerizado de imágenes AFM, Porometría de aire-líquido, Porosimetría de mercurio

\section{Characterization of a new ceramic membrane of microfiltration with a flexible stainless steel metallic support}

In this work we present the characterization of a ceramic flexible membrane, made by Synthesechemie ${ }^{\circledR}$, with a nominal pore size of $0.45 \mu \mathrm{m}$. This membrane consists in an $\alpha$-alúmina porous support which has an embedded stainless steel metallic network, onto what a selective layer of titanium dioxide has been deposited.

Membrane characterization has been realized with the aid of several techniques as: atomic force microscopy (AFM), air-liquid displacemente porosimetry and mercury porosimetry. Each tecnique giving information on different aspects: surface characteristics, bulk pores and active pores. Although the total pore size distribution is rather broad, the active pores (those opened to flux) present a narrow distribution with a mean pore size very close to the manufacturer nominal value.

Key words: Porous materials, Membranes, Computerized analysis of AFM images, Air liquid porometry, Mercury porosimetry

\section{INTRODUCCIÓN}

La utilización de membranas inorgánicas en procesos de separación está creciendo de forma continuada. Entre las principales ventajas que dichas membranas (y, en especial las membranas cerámicas) presentan está su gran estabilidad frente a todo tipo de condiciones extremas. Así suelen ser membranas estables frente a todo tipo de disolventes, pueden trabajar a valores de $\mathrm{pH}$ extremos y a temperaturas elevadas (con la posibilidad inherente de ser esterilizas). Ello las hace especialmente útiles en procesos en los que se busca la separación de componentes de interés industrial en fluidos de alimentación con compuestos peligrosos o medio-ambientalmente nocivos.

Por otro lado, la optimización de una membrana comercial para los procesos en los que presente mayor efectividad, es un aspecto de importancia relevante que, en último término, pasa por poseer un conjunto de técnicas de caracterización que permitan el mas exhaustivo y exacto conocimiento previo de la membrana.

Existen diversos métodos de caracterización de membranas, los cuales permiten determinar la distribución de tamaños de poro presentes en la membrana, (1-5). Algunos de ellos sólo pueden determinar la distribución de poros activos, mientras que otros no permiten distinguir entre poros activos o no activos. Entre los métodos que pueden detectar sola- mente poros activos, mencionaremos únicamente el método del punto de burbuja, introducido por Bechhold, (6). Esta técnica, se basa en la determinación de la presión necesaria para hacer pasar un fluido a través de una membrana porosa cuyos poros están ocupados por algún líquido. Los métodos de puntos de burbuja y métodos relacionados (como los de desplazamiento de líquido-gas y de líquido-líquido) se usan frecuentemente para la determinación del tamaño de poro medio así como de las distribuciones de tamaños de poro de muchas membranas comerciales, (7-10), por lo cual han adquirido el estatus de métodos estándar recomendados $(\mathbf{1 1}, \mathbf{1 2})$.

Las técnicas microscópicas son, en principio, solamente capaces de caracterizar la superficie de la membrana. Sin embargo, dado que los poros activos deberían ser los únicos presentes en la superficie de la membrana del lado de la alimentación, estas técnicas pueden ser útiles para dar información principalmente sobre poros activos. Es sin embargo claro que el uso de cortes transversales de la membrana podría permitirnos obtener información sobre el resto de poros en principio no-activos. Entre estas técnicas, la más novedosa es la de microscopía de fuerza atómica (AFM). Esta técnica permite el estudio de la superficie de materiales no-conductores, llegando a una resolución en la escala de los angströms. Desarrollada por Binnig y colaboradores, (13), su principal 
ventaja sobre las técnicas de microscopía electrónica es que no requiere ninguna preparación previa de la muestra, (14). Aún siendo una técnica relativamente novedosa, su aplicación al campo de la caracterización de membranas, tanto biológicas como sintéticas, está creciendo rápidamente, $(\mathbf{1 5}, \mathbf{1 6})$.

Finalmente entre las técnicas que detectan todos los poros, tanto activos como no activos, podemos mencionar la porosimetría de mercurio. Su fundamento es el mismo que el del método del punto de burbuja, sin embargo en este caso es el mercurio (un fluido no mojante) el que es introducido en los poros de una membrana seca, (17). En este caso es importante tener en cuenta que para introducir el mercurio en los poros mas pequeños se hace necesario usar presiones extremadamente altas, lo cual puede inducir deformaciones en la estructura de los poros.

\section{MÉTODOS DE CARACTERIZACIÓN ESTRUCTURAL}

\subsection{Microscopía de Fuerza Atómica}

La microscopía de fuerza atómica, presenta interesantes posibilidades de aplicación en el estudio de filtros de membrana, permitiendo el estudio superficial de materiales noconductores (como de hecho es lo normal encontrar en el campo de fabricación de membranas) hasta la escala del nanómetro. Su principal ventaja sobre las técnicas microscópicas habituales (microscopía electrónica, principalmente) es que, al no necesitar ningún tipo de preparación previa de la muestra, no se inducen errores debidos al propio método en los resultados.

En este trabajo se han realizado experiencias de Microscopía de Fuerza Atómica, (AFM), utilizando el Modo Tapping ${ }^{\circledR}$, con un equipo Nanoscope IIIA, de Digital Inst. ${ }^{\circledR}$. En este modo de operación, la punta y el brazo flexible (cantiléver) que la soporta son hechos oscilar a una frecuencia cercana a su frecuencia de resonancia. En este caso no se mide la amplitud de la deflexión del cantiléver (como ocurre en otras técnicas convencionales de AFM: tanto los métodos de contacto como de no-contacto), sino que se determina la raíz cuadrática media de la intensidad del rayo láser después de ser reflejado por el cantiléver. Esta técnica es especialmente útil debido a que limita la posibilidad de daños tanto en la punta de barrido como en la muestra, debido a que el contacto entre ambos es intermitente, dando por otro lado imágenes de mejor calidad que las obtenidas en el modo no-contacto, así como libres de los errores que frecuentemente aparecen cuando se usan las técnicas convencionales. Las puntas usadas en el trabajo consisten en un monocristal de silicio con forma cónica y un ángulo en la punta inferior a $15^{\circ}$ y un radio de curvatura menor de $20 \mathrm{~nm}$. Las imágenes fueron obtenidas a temperatura ambiente y las áreas de barrido en las imágenes se escogieron de forma que se tuviera una buena definición al tiempo que un número razonable de poros a fin de realizar el correspondiente tratamiento estadístico.

El análisis de imagen computerizado se realizó mediante un software Jandel ${ }^{\circledR}$ ScanPro (version 3.00.0030). Cada fotografía fue digitalizada con una resolución de 510 x 514 pixels, asignando un nivel de gris entre 0 (negro) y 255 (blanco). Luego se realizó un equalización y se eligió un nivel de gris umbral por debajo del cual se acepta que todo pixel corresponde a un poro, (18).Con el fin de evitar los efectos de la iluminación desigual, se procedió a eliminarlos dividiendo la imagen en rectángulos iguales y asignando a todos los pixels en cada recuadro una intensidad tal que los pixels originales tengan superior intensidad en un $95 \%$. Finalmente por medio de interpolación lineal se construye una nueva imagen que se sustrae de la original.

\subsection{Porometría Aire-Líquido}

Las determinaciones de porosimetría de aire-líquido se realizaron en un equipo Coulter ${ }^{\circledR}$ Porometer II, manufacturado por Coulter Electronics Ltd. Este aparato usa la técnica de desplazamiento de líquidos. Para ello la muestra es completamente empapada en un líquido (Coulter ${ }^{\circledR}$ Porofil) de baja tensión superficial $\left(\gamma=16 \cdot 10^{-3} \mathrm{~N} / \mathrm{m}\right)$, baja presión de vapor $\mathrm{mm} \mathrm{Hg}$ a $298 \mathrm{~K}$ ) y baja reactividad. Se asume que el líquido rellena la totalidad de los poros presentes en la membrana, dado que tiene un ángulo de contacto prácticamente nulo con la casi totalidad de materiales usualmente encontrados en tecnología de membranas. A la muestra húmeda se le aplican entonces presiones crecientes a través de una fuente de aire limpio y seco, a $313 \mathrm{~K}$. Así al aumentar la presión del aire se alcanza un punto en el que dicha presión es suficiente para sobrepasar la tensión superficial del líquido en el interior de los poros más grandes, de forma que el líquido es expulsado. Si continuamos incrementando la presión, se irán vaciando poros de tamaños cada vez más pequeños a través de los cuales comenzará a fluir aire, de forma que un análisis del flujo de aire en función de la presión nos permita detectar la presencia de poros comprendidos entre 0,05 y $300 \mu \mathrm{m}$.

\subsection{Porosimetría de Intrusión de Mercurio}

Se ha utilizado un porosímetro de mercurio Autopore III 9410 de Micromeritics ${ }^{\circledR}$. Este equipo permite el análisis de la distribución de tamaños de poro así como su contribución a la porosidad total, para poros comprendidos en el rango de 0.003 a $360 \mu \mathrm{m}$, lo cual corresponde a presiones aplicadas de hasta 60,000 psia. (414-106 Pa). El rango de trabajo elegido es analizado de forma automática por el equipo una vez alcanzadas las condiciones estáticas para cada presión o tamaño de

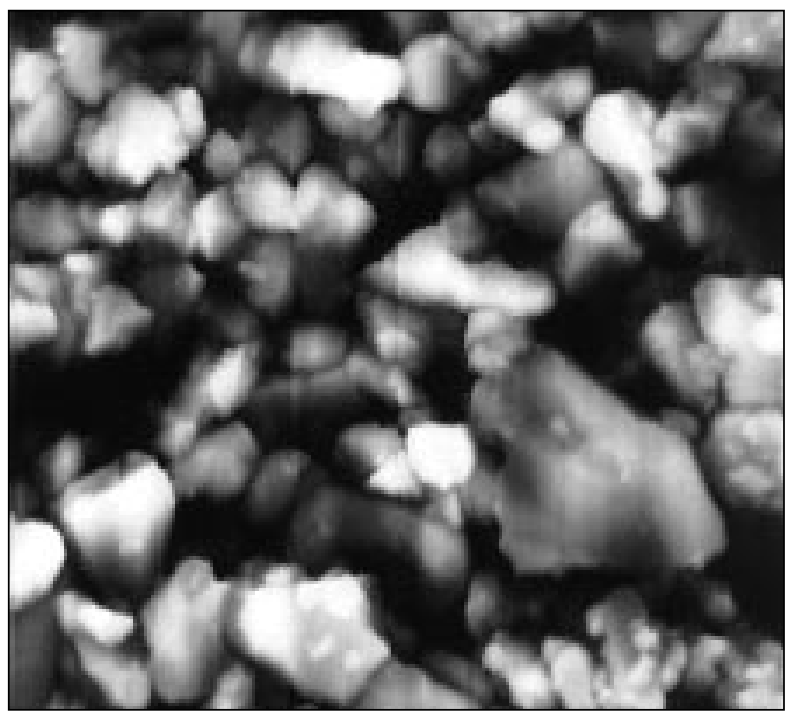

Fig. 1.- Imagen de la superficie obtenida por microscopía de fuerza atómica (AFM), utilizando la técnica de contacto intermitente. 
poro equivalente. Es imprescindible un conocimiento lo más exacto posible del ángulo de contacto mercurio-material de membrana. Este ángulo podría ser medido directamente sin embargo, para ello sería preciso disponer de láminas totalmente planas hechas del mismo material que el que forma la membrana. Como eso es imposible en la mayoria de los casos, lo que se hace es utilizar un valor estándard que se ha mostrado válido para una gran cantidad de membranas de análogas características en este caso se ha utilizado un valor de $130^{\circ},(5,17,19)$.

\section{MATERIALES Y MÉTODOS EXPERIMENTALES}

En este trabajo, se ha utilizado una membrana de microfiltración de reciente comercialización. Se trata de una membrana cerámica de $\alpha$-alúmina sobre la cual se ha depositado una capa fina de óxido de titanio, fabricada por Synthesechemie $\mathrm{GmbH}$, y con un diámetro de poro nominal de $0.45 \mu \mathrm{m}$. La membrana ha sido producida por una modificación de la técnica sol-gel, unido a un calentamiento rápido para dar consistencia a la capa fina selectiva. El soporte de $\alpha$-alúmina está a su vez soportado por un tejido de hilo de acero inoxidable, a fin de darle suficiente estabilidad y flexibilidad. Con estas características la membrana es estable frente a la mayor parte de los disolventes, así como a rangos extremos de $\mathrm{pH}$, presentando una temperatura máxima de trabajo de $650^{\circ} \mathrm{C}$. En principio presenta posibles aplicaciones en separación de gases, pervaporación y otros procesos similares.

\section{RESULTADOS}

Comenzando por un estudio superficial de las membranas se realizaron diversas imágenes de Microscopía de Fuerza Atómica, según la técnica descrita. Un ejemplo se presenta en la Fig. 1, donde se muestra una imagen de la superficie activa de la membrana, sobre un área escaneada de 3.5 micras. En la

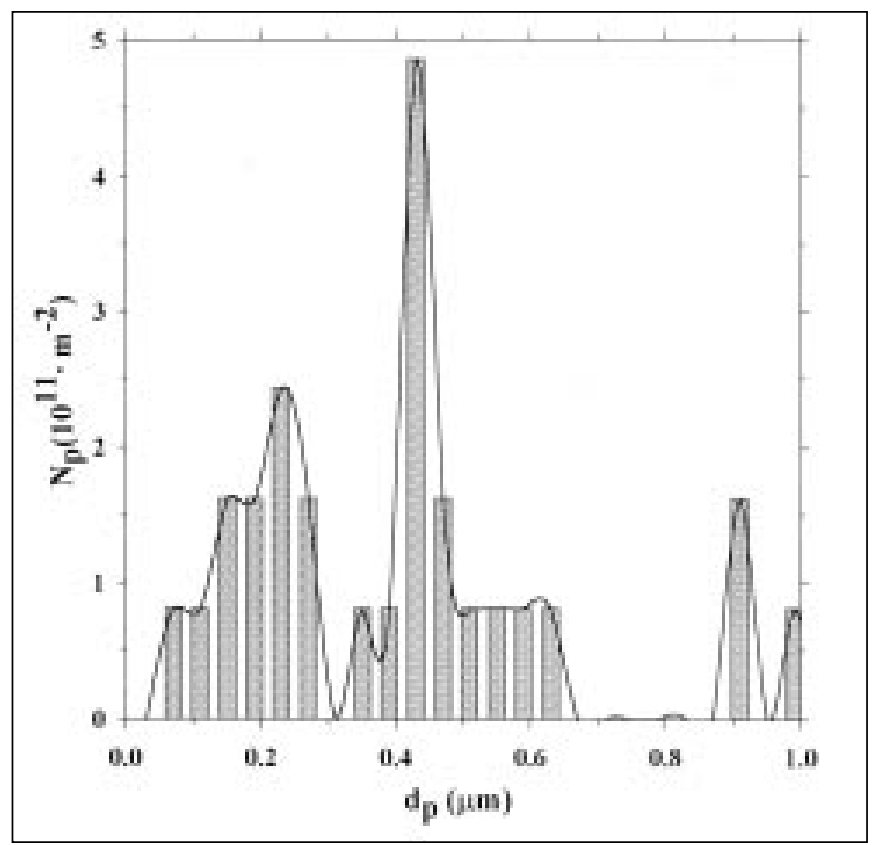

Fig. 2.- Densidad de poros obtenida por análisis de imagen computerizado sobre las figuras de AFM. imagen se observa claramente la existencia de una distribución amplia de tamaños en las partículas de óxido de titanio que, como ya comentamos, forman el recubrimiento superior de la membrana. En una primera inspección se puede observar como existen partículas de tamaño superior a $1 \mu \mathrm{m}$ junto a otras que apenas alcanzan la décima parte de ese tamaño.

Para aclarar la existencia de esta distribución se procedió a realizar un completo análisis de imagen sobre un conjunto suficiente de fotografías de manera que los resultados presentarán una razonable fiabilidad estadística. El resultado de este análisis se presenta en la Fig. 2, donde se observa claramente la amplitud ya comentada de la distribución de tamaños de los huecos que dejan entre si las partículas de óxido de titanio. No obstante se observa claramente un pico de altura superior a todos los demás, centrado en valores muy cercanos a los nominales.

Por otro lado, cuando se analizan los resultados de porosimetría de mercurio, esta amplitud de la distribución se hace

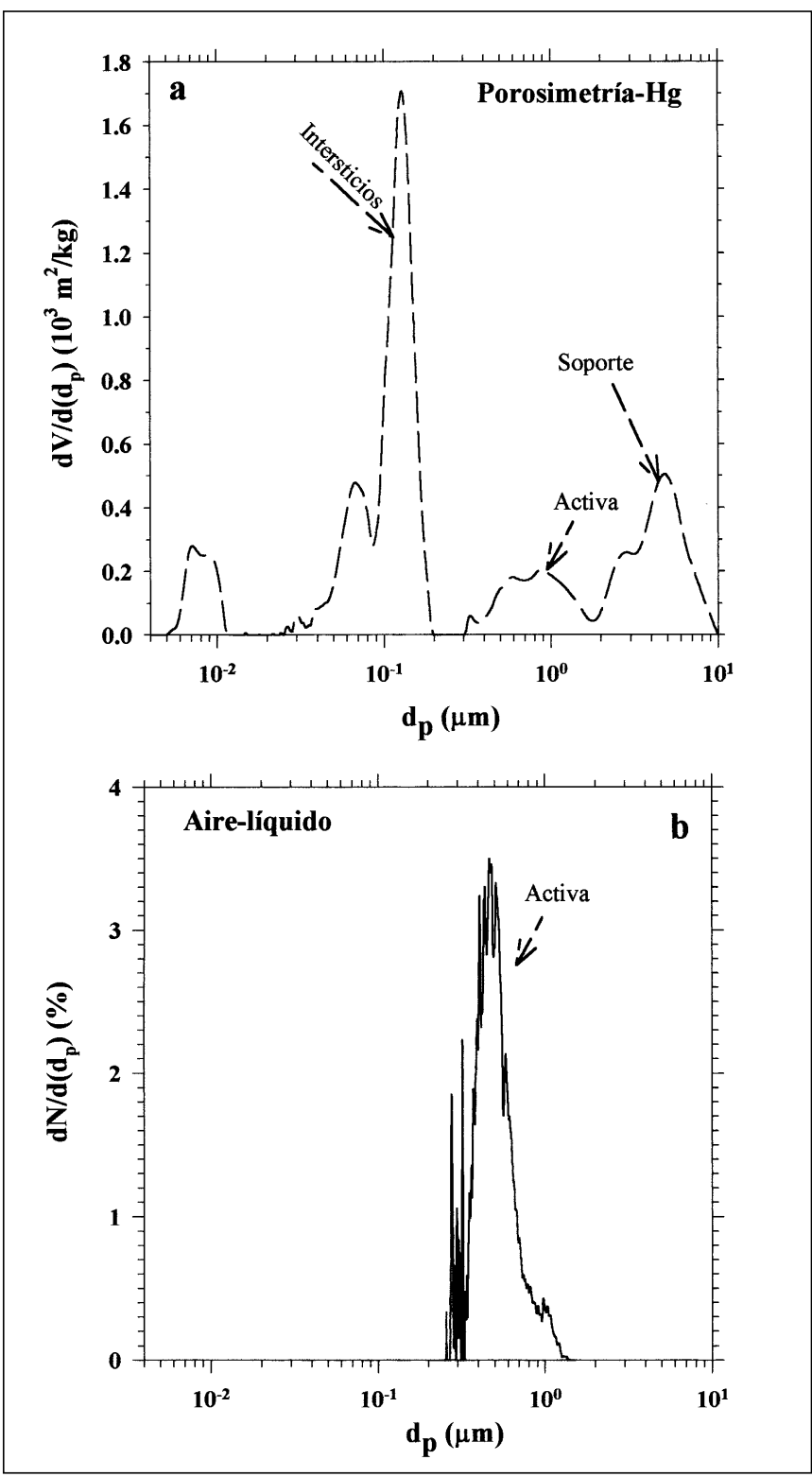

Fig. 3.- Distribución diferencial de volúmenes de poro obtenida por porosimetría de mercurio (3.a) y la distribución diferencial de números de poros obtenida mediante porometría de desplazamiento airelíquido (3.b). 
incluso mayor. En efecto, en la Fig. 3, se observa claramente como dicha distribución se extiende a valores de tamaño de poro ampliamente superiores a los nominales. Asimismo, es sabido que la porosimetría de mercurio no es capaz de distinguir entre poros superficiales y los presentes en el interior de la membrana, ya que detecta la totalidad de los poros presentes en la muestra. En consecuencia, si se comparan los resultados de AFM con los de intrusión de mercurio, resulta evidente que estos grandes poros presentes en este último análisis han de estar situados en el interior del volumen de la membrana, más bien que en la superficie, correspondiendo, con toda probabilidad al soporte de $\alpha$-alúmina sobre el que se depositó el óxido de titanio.

Es evidente, además, que ambas técnicas son capaces de detectar tanto poros activos (i.e., abiertos al flujo) como poros no activos, de forma que si se quiere tener un conocimiento sobre la distribución de poros realmente pertinentes en cuanto a las propiedades de permeación de la membrana, hay que combinar estos resultados con otras técnicas de caracterización que nos den este tipo de información. En nuestro caso, dado el rango de poros con el cual estamos tratando, hemos realizado este análisis por medio de la porometría de desplazamiento de líquidos. Esta técnica, dadas sus características, ya descritas, detecta únicamente los poros efectivamente abiertos al flujo a través de la membrana. De esta forma se ha obtenido la distribución que se muestra, junto a los resultados de porosimetría de mercurio, en las Fig. 3.a y 3.b. Ahora es claro como, aunque las distribuciones de poros totales (tanto la de superficie, ver Fig. 2; como la de volumen, en la misma Fig. 3.a) son amplias por contener poros de diversas características y estructura, los poros activos presentan una distribución bastante estrecha y centrada muy aproximadamente en los valores nominales.

\section{CONCLUSIONES}

Para terminar el análisis de los resultados obtenidos, podemos realizar una comparación conjunta de los resultados de las tres técnicas (comparación que no se ha realizado en una misma figura para mayor claridad de los resultados presentados), la cual nos permite extraer interesantes conclusiones sobre la situación de los poros encontrados en cada distribución.

A) En primer lugar se puede concluir que los poros activos corresponden a poros presentes en la superficie activa de la membrana, como se puede deducir del hecho de que el pico máximo de análisis de imagen (ver Fig.2) coincide prácticamente con la distribución obtenida por porosimetría de desplazamiento de aire-líquido (ver Fig. 3.b).

B) No obstante existen en la distribución de superficie algunos poros que no se encuentran en la porosimetría de aireliquido, debiéndoseles por tanto considerar como poros no activos. Dichos poros, que por otro lado se encuentran también representados en la distribución por porosimetría de mercurio (Fig. 3.a), puede suponerse que son intersticios o huecos que quedan entre las partículas de óxido de titanio que conforman la capa selectiva. Dichos intersticios no llegan a penetrar totalmente la capa activa, por lo cual no contribuyen al flujo a través de la membrana.

C) Finalmente, los poros de tamaños mayores a $1 \mu \mathrm{m}$, que aparecen principalmente en los resultados de porosimetría de mercurio (con lo cual hemos de considerarlos como poros existentes en el interior del volumen de la membrana, Fig. 3.a) se pueden asimilar a poros del soporte de la membrana.

En resumen se ha mostrado como la utilización conjunta de diversas técnicas de caracterización estructural como las aquí consideradas, nos permite obtener una imagen completa de la estructura real de la membrana. Esto resulta imprescindible con el fin de prever su utilidad en los procesos de separación por membrana. En este sentido la combinación de técnicas detectoras de poros activos, junto a aquellas técnicas que detectan la totalidad de los poros, bien en superficie o en volumen, nos da una mayor información así como una visión realista sobre la estructura de la membrana analizada.

\section{AGRADECIMIENTOS}

Los autores desean agradecer a Syntesechemie $\mathrm{GmbH}$, la amable cesión de muestras de su membrana de microfiltración. El trabajo realizado ha sido financiado con cargo a los siguientes proyectos de Investigación: QUI96-0767 del "Plan Nacional de Investigación y Desarrollo" (CICYT) y VA10496 de la Junta de Castilla y León.

\section{REFERENCIAS}

1. M. Mulder, Basic Principles of Membrane Technology, Kluwer, Dordrecht, The Netherlands, 1991, 110-144.

2. K. Kamide and S. Manabe, in Ultrafiltration Membranes and Applications, A.R. Cooper (Ed.), Plenum Press, New York, NY, USA, 1980, 173-202.

3. B. Rasneur, Porosimetry (Characterization of Porous Membranes), Summer School on Membr. Sci. and Tech., Cadarache, France, 1984.

4. R.E. Kesting, Synthetic Polymeric Membranes, A Structural Perspective, 2nd. ed., John Wiley and Sons, New York, NY, USA, 1985, 1-21.

5. S. Lowell and J.E. Shields, Powder Surface Area and Porosity, Powder Technology Series, B. Scarlett (Ser.Ed.), J. Wiley and Sons, New York, NY, USA, 1987, 97-120.

6. H. Bechhold, M. Schlesinger, K. Silbereisen, L. Maier and W. Nurnberger, "Pore diameters of ultrafilters", Kolloid Z., 55172 (1931).

7. K. Schneider, W. Hölz, R. Wollbeck and S. Ripperger, "Membranes and modules for transmembrane distillation", J. Memb. Sci., 3925 (1988).

8. G. Reichelt, "Bubble point measurements on large areas of microporous membranes", J. Memb. Sci., 60253 (1991).

9. L. Zeman, "Characterization of microfiltration membranes by image analysis of electron micrographs. Part. II. Functional and morphological parameters", J. Memb. Sci., 71233 (1992).

10. A. Hernández, J.I. Calvo, P. Prádanos and F. Tejerina, “Pore size distributions in microporous membranes. A critical analysis of the bubble point extended method", J. Memb. Sci., 1121 (1996).

11. ASTM F316, Standard Test Method for Pore Size Characteristics of Membrane Filters by Bubble Point and Mean Flow Pore Test.

12. ASTM E1294, Standard Test Methods for Pore Size Characteristics of Membrane Filters Using Automated Liquid Porosimeter.

13. G. Binnig, C.F. Quate and Ch. Gerber, "Atomic force microscopy”, Phys. Rev. Lett., 56930 (1986).

14.S. Nakao, "Determination of pore size and pore size distribuion. 3. Filtration membranes", J. Memb. Sci, 96131 (1994).

15. A. K. Fritzsche, A.R. Arevalo, M.D. Moore, C.J. Weber, V.B. Elings, K. Kjoller and C.M. Wu, "The surface structure and morphology of polyvinylidene fluoride microfiltration membranes by atomic force microscopy", J. Memb. Sci, 6865 (1992).

16.P. Dietz, P.K. Hansma, O. Inacker, H.D. Lehmann and K.H. Herrmann, "Surface pore structures of micro- and ultrafiltration membranes imaged with the atomic force microscope", J. Memb. Sci., 65101 (1992).

17.E. Honold and E.L. Skau, "Applications of mercury intrusion method for determination of pore-size distribution of membrane filters", Science, 120 805 (1954).

18. A. Hernández, J.I. Calvo, P. Prádanos, L. Palacio, M.L. Rodríguez and J.A. de Saja, "Surface structure of microporous membranes by computerized SEM image analysis applied to Anopore filters", J. Memb. Sci., 13789 (1997).

19. T. Allen, Particle Size Measurement, v.1, Chapman and Hall, London, 1997, 149-190.

Recibido: $21-10-98$

Aceptado: 8-2-99 